\title{
Space Radiation Measurement on the Polar Route onboard the Korean Commercial Flights
}

\author{
Junga Hwang ${ }^{1 \dagger}$, Jaejin Lee ${ }^{1}$, Kyung-Suk Cho ${ }^{1}$, Ho-Sung Choi ${ }^{1,2}$, \\ Su-ryun Rho ${ }^{1}$, and Il-Hyun Cho ${ }^{1}$ \\ ${ }^{1}$ Solar and Space Weather Research Group, KASI, Daejeon 305-348, Korea \\ ${ }^{2}$ University of Science and Technology, 113 Gwahangno, Yuseong-gu, Daejeon 305-333, Korea \\ E-mail: jahwang@kasi.re.kr
}

(Received February 1, 2010; Accepted February 24, 2010)

\begin{abstract}
This study was performed by the policy research project of Ministry of Land, Transport and Maritime Affairs, which title is "Developing safety standards and management of space radiation on the polar route". In this research, total six experiments were performed using Korean commercial flights (B747). Three of those are on the polar route and the other three are on the north pacific route. Space radiation exposure measured on the polar route is the average $84.7 \mathrm{uSv}$. The simulation result using CARI-6M program gives $84.9 \mathrm{uSv}$, which is very similar to measured value. For the departure flight using the north pacific route, the measured space radiation is the average $74.4 \mathrm{uSv}$. It seems that is not so different to use the polar route or not for the return flight because the higher latitude effect causing the increase of space radiation is compensated by the shortened flight time effect causing decreasing space radiation exposure.
\end{abstract}

Keywords: space radiation, polar route, space weather

\section{Introduction}

Space radiation measurement experiments are vital for flight attendants and flight passengers in order to correctly understand space radiation exposure from aircraft flying at different altitudes. Space radiation is a by-product of interaction between radioactive sources from space and the earths atmosphere. The general term, Cosmic Radiation, refers to high-energy particles and gamma rays falling from space to the earth; among which are high-energy particles, as well as gamma rays developed secondarily due to collisions with molecules in the atmosphere. Space radiation can be dvided by primary space radiation which is prior to collisions with molecules in the atmosphere and secondary space radiation made by collisions with air particles and primary particles. A primary space radiation is redefined as GCR (Galactic Cosmic Radiation), coming from outside the solar system, and SCR (Solar Cosmic Radiation), attributed to sunspot activities of the solar system. GCR is generated as a result of Coronal Mass Ejection, Supernova Explosion, Pulsar Acceleration, and Galactic Nuclei Explosion, and is comprised of $95 \%$ of proton, $3.5 \%$ of an alpha particle,

\footnotetext{
${ }^{\dagger}$ corresponding author
} 


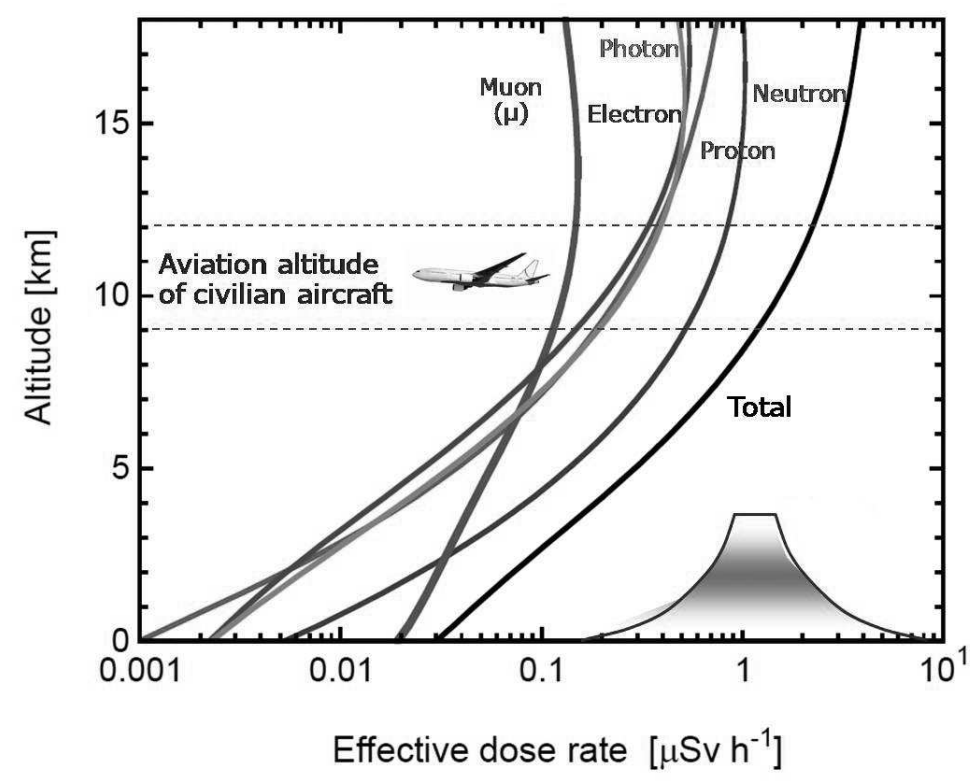

Figure 1. Effective dose rate depending on the altitude.

Table 1. Comparison between medical and space radiation exposure.

\begin{tabular}{lc}
\hline \hline \multicolumn{1}{c}{ Item } & Total dose (uSv) \\
\hline Chest x-ray & 20 \\
Dental x-ray & 1 \\
Polar route(ICN-NY) & $85^{*}$ \\
\hline
\end{tabular}

and rest with heavy ions, such as carbon and iron. After GCR is generated, it is accelerated with high-energy $\left(\sim 10^{20} \mathrm{eV}\right)$ particles and reaches the earths atmosphere. Meanwhile, SCR is space radiation occurred based on sunspot activities, reaches maximum energy of $10^{9} \mathrm{eV}$, and is comprised of muons, electron, protons, photons, and neutrons (Lewis et al. 1999). It is known that the presence of muons is the highest rate in aircraft altitudes, followed by electron protons, and photons in similar rate, and neutrons show relatively low presence.

As shown in Figure 1, space radiation in flight altitudes display a different aspect compare to the distribution demonstrated in the earth. In a flight altitude, the composition and strength of space radiation change depending intensity and altitude of a primary space radiation input into the atmosphere, and the intensity of primary spacecraft is affected by solar activities and earths magnetic field. The space radiation in flight altitudes, therefore, can be referred to as a function in accordance with the solar activity and magnetic field, namely, (1) altitude, (2) latitude, and (3) solar activities.

To accurately confirm the type and energy of radiation in flight altitudes, various types of radiation measuring equipment are used. Although the sample was not conducted based on many numbers of flight attendants, the fact that the frequency of biological damage is higher in the samples exposed to space radiation in flight altitudes supports the argue that space radiation exposure is different from that of the earth (Reynolds et al. 2002, Cavallo et al. 2002). Table 1 is a comparison 


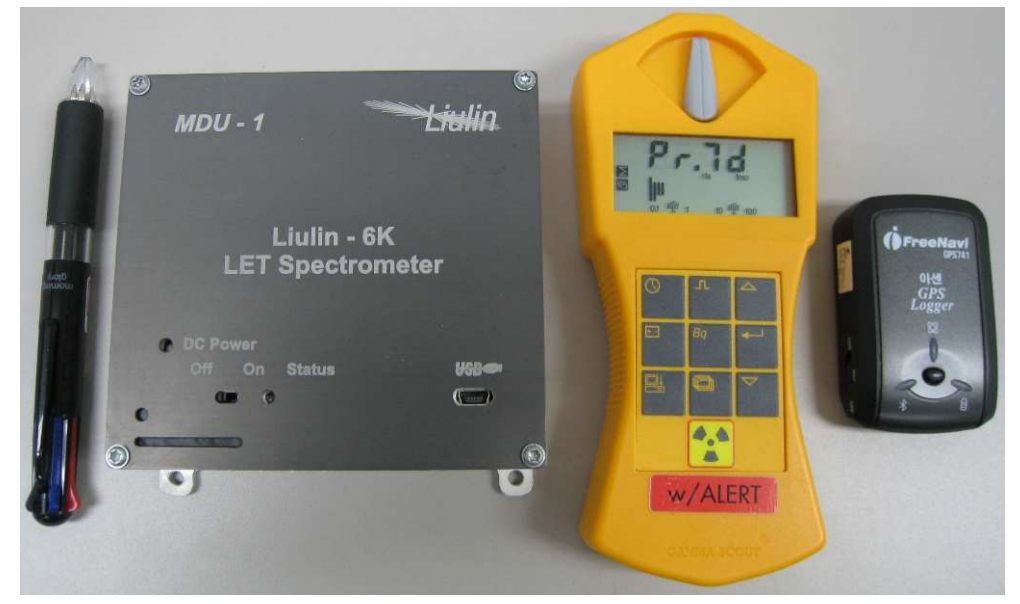

Figure 2. Measuring equipment for the space radiation, from left to right, Liulin-6K LET spectrometer, gamma scout, and GPS logger.

illustrating medical radiation exposure, which is widely available for a living environment against space radiation exposure in one time air travel. As the result shown in Table 1, space radiation exposure is definitely not a small value compared to medical radiation exposure. Therefore, efforts to change flight schedules are crucial to control exposure of space radiation. For such experiments, an adequate radiation exposure limit should be suggested, and precise practical measurement experiments should be conducted in advance regarding radiation exposure level for flight attendants. As part of efforts, the study has been proceed as a task of the Office of Civil Aviation of the Ministry of Land, Transport and Maritime Affairs in 2009, which title is "Developing safety standards and management policy of space radiation on the polar route", and conducted in the process of practical measurement on space radiation in domestic commercial flights.

\section{Experiments}

The radiation measurement equipment used for measurement experiments is as Figure 2. In the order from left: Liulin-6K spectrometer is widely used for a simple purpose to measure space radiation in flight altitudes, gamma-scout counter for radiation measurement on the earth, and GPS logger to obtain precise latitude and altitude information. Liulin-6K spectrometer is designed to be onboard by considering a radiation source in flight altitudes from STIL-BAS (Solar-Terrestrial Influences Laboratory - Bulgarian Academy of Sciences), and is space radiation measurement equipment allowing constant monitoring of a total radiation exposure and energy spectrum. Space radiation measurement experiments in flight altitudes using Liulin have been already implemented in various commercial aircrafts and ISS (International Space Station), and the reliability has been proven (Spurny \& Dachev 2003, Spurny et al. 2004, Green et al. 2005). Liulin-6K is designed to be operated by $28 \mathrm{~V} \mathrm{DC}$, which can be supplied from an aircraft or lithium-ion battery. An insulated DC/DC converter is crafted to meet the safety requirements of aircraft regulations. It can be used for 60 days consecutively if operated by battery only. A spectrometer is comprised of a semiconductor detector $\left(2 \mathrm{~cm}^{2}\right.$ area, $0.3 \mathrm{~mm}$ thickness), a charge-sensitive preamplifier, two micro-controllers, 1GB flash memory and a USB bridge. The actual size is $110 \times 100 \times 45 \mathrm{~mm}$, and the weight is $0.57 \mathrm{~kg}$. 


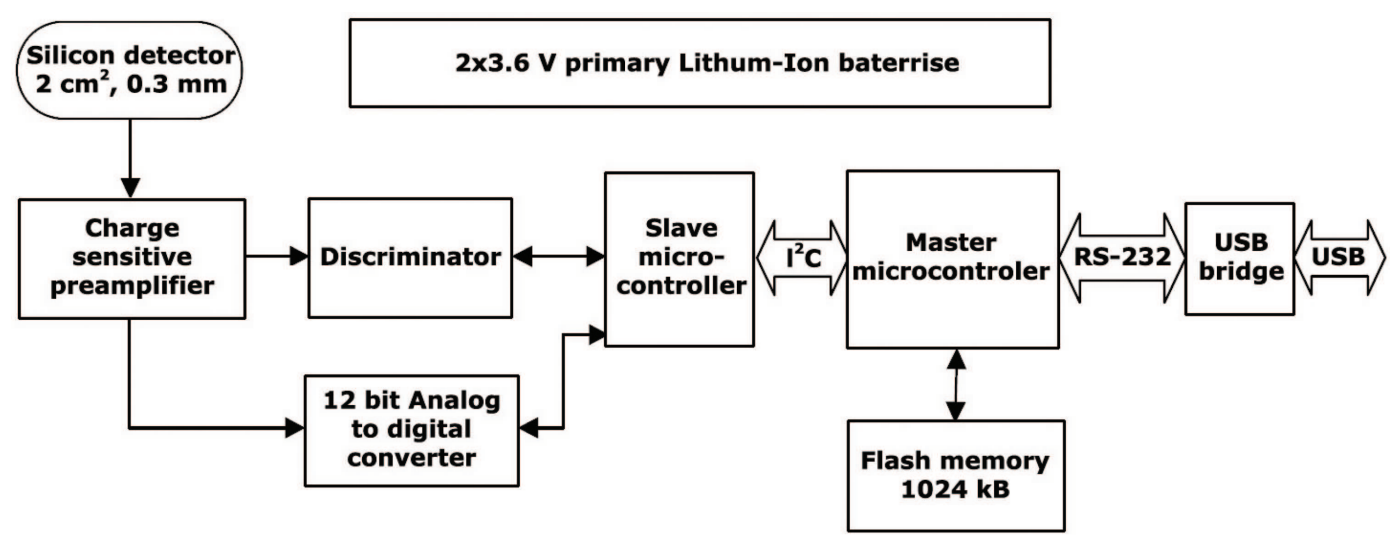

Figure 3. Block diagram of Liulin-6K spectrometer.

As shown in Figure 3, it could be simple to conduct an experiment in a cabin if power is supplied through two 3.6V lithium-ion batteries. Thus, Liulin-6K has many advantages, such as its compact size, light weight and long-lasting power. The gamma-scout used in experiments response to proton, electron, and gamma rays, but not at a neutron. It also has a weak point that it can only observe under $2 \mathrm{MeV}$ radiation, and is not a proper device for aircraft radiation measurement. However, an experiment is conducted with the device onboard to compare overall total exposure of space radiation. For a comparison with measured value, the CARI-6M program provided by FAA (Federal Aviation Administration) is used for space radiation calculation. The CARI-6M is an enhanced program that allows altitude, latitude, and longitude information input according to aviation routes. The CARI-6 program uses flight dates, departure and arrival airports, and route information (altitude, flight time), and calculates a cumulative dose based on dose rate data according to LUIN99 and LUIN2000. A desired route can be selected as international Civil Aviation Organization (ICAO) airport code is input, and those airports not registered in the device can be input separately. Select a departure and arrival airport and provide a flight altitude, calculate exposure based on the presumption that an aircraft has moved following a specific route, which is calculated in a great circle.

\section{Analysis}

The radiation measurement equipment shown in Figure 2 was on Korean commercial flights, and a total of six measurements were conducted. Total six radiation measurements were implemented: three times on departure flights and the other three times on returning flights. The three measurements on an returning flight was a New York-Incheon route in which used the polar route, and the other three measurements were an Incheon-New York route, which used the north pacific route. Figure 4 is the mark on the three-dimensional Google map provided based on the data of GPS logger measured along with actual flight route at the time. The first experiment was conducted on October 7, using a B747 aircraft of A airline in Korea is the space radiation measured in the polar route, and the result is as Figure 5. In Figure 5, the square symboled line represents a Liulin-6K spectrometer, the solid line is gamma-scout, and the dotted lines refer to altitude information. As shown, the value measured with Liulin-6K changes up to $4 \sim 10 \mathrm{uSv} / \mathrm{hr}$, while gamma-scout is $2 \sim 3 \mathrm{uSv} / \mathrm{hr}$, which 


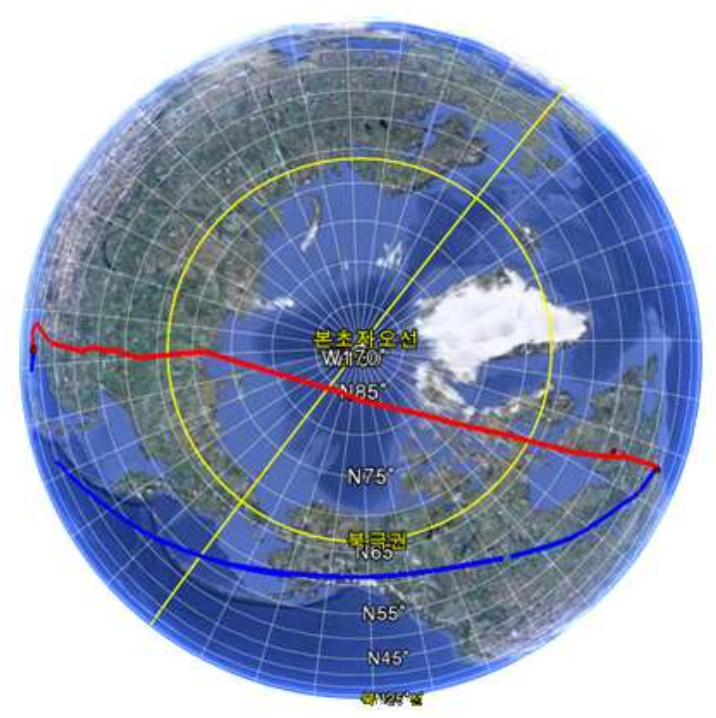

Figure 4. Comparison between the polar route (red) and the north pacific route (blue).

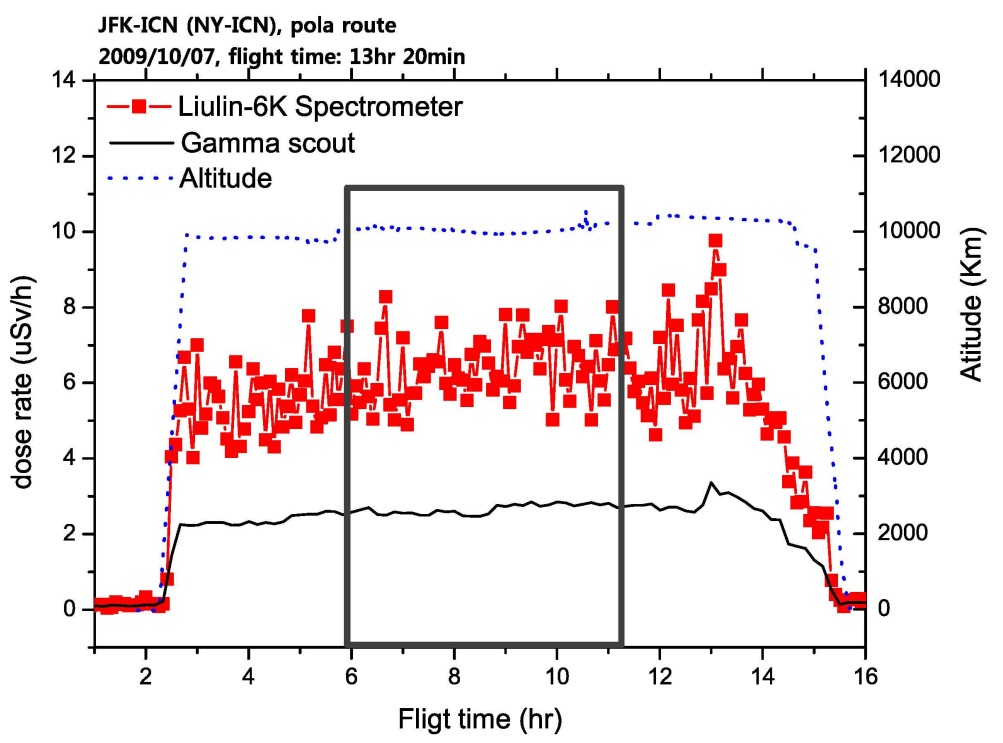

Figure 5. The first experiment measuring the space radiation dose of the polar route (A airline).

is only $1 / 3$ of the result value of Liulin-6K. That is, general equipment that measures radiation on the earth cannot measure space radiation exposure in all. We could probably assume that the impact of space environment and latitude can be a cause that develops a change in exposure affected per 


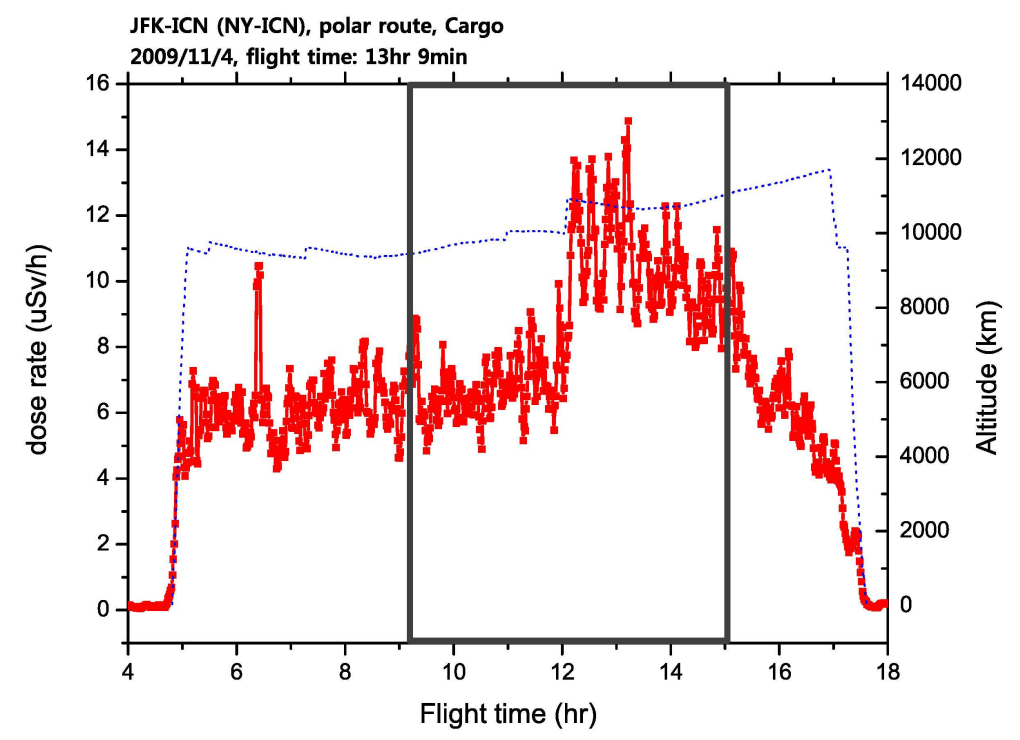

Figure 6. The second experiment measuring the space radiation dose of the polar route (B airline cargo).

hour up to $4 \sim 10 \mathrm{uSv} / \mathrm{hr}$ in a region with constant region. Additionally, since an time resolution of Liulin-6K was five minute intervals, the value seems to increase discontinuously in a conversion process per hour. Figure 5 illustrates the cumulative radiation exposure measured via Liulin-6K, for 13 hours and 20 minutes of total flight time, was $76.07 \mathrm{uSv}$. A calculated value of CARI-6M, which was based on measured GPS data was $63 \mathrm{uSv}$, showing a $17 \%$ less value than the actual measurement value. The difference in measured value and the calculated result of CARI-6M seems that while CARI-6M approximately calculates space radiation with several location information and intermediate points, a precise value is displayed each time the measurement is conducted, which result in demonstrating such a difference. The average exposure was $4.70 \mathrm{uSv} / \mathrm{hr}$, while peak exposure was $9.76 \mathrm{uSv} / \mathrm{hr}$. Figure 5 shows the actual flight time in the polar circle (latitude more than 65 degree), which is marked with a thick rectangle mark. The actual flight time in the polar circle is approximately 5 hours and 40 minutes. The average exposure was $6.38 \mathrm{uSv} / \mathrm{hr}$, which is relatively a higher value compare to $4.70 \mathrm{uSv} / \mathrm{hr}$, an average exposure of the total flight time. When compared with average exposure $3.45 \mathrm{uSv} / \mathrm{hr}$, at the time not passing (latitude under 65 degree) the polar circle, the exposure result obtained when actually passing the polar circle has increased by two fold in dose rate. However, the total cumulative dose during 5 hours and 40 minutes, which was actual flight time on the polar route, was $34.56 \mathrm{uSv}$, accounting about $45 \%$ of a total exposure of 76.07. Comparing only the average exposure from the top of the polar circle and cumulative exposure for 13 hours and 20 minutes of total flight time, the exposure from the polar route showed higher result. However, the cumulative exposure (34.56 uSv) of the polar route showed a similar result to that of the rate $(42.5$ $\%$ ) of the time on the polar route (5 hours and 40 minutes) over the total flight time (13 hours and 20 minutes). That is, we can assume that no so much space radiation will be accumulated when flying the polar route. The occurrence of radiation increases around 13:00 hours; this can be put down to the cumulative effect resulting from momentary increases in attitude of more than $10 \mathrm{~km}$. Additionally, the reason for space radiation decrease around 14 hours despite altitude increase is presumably 


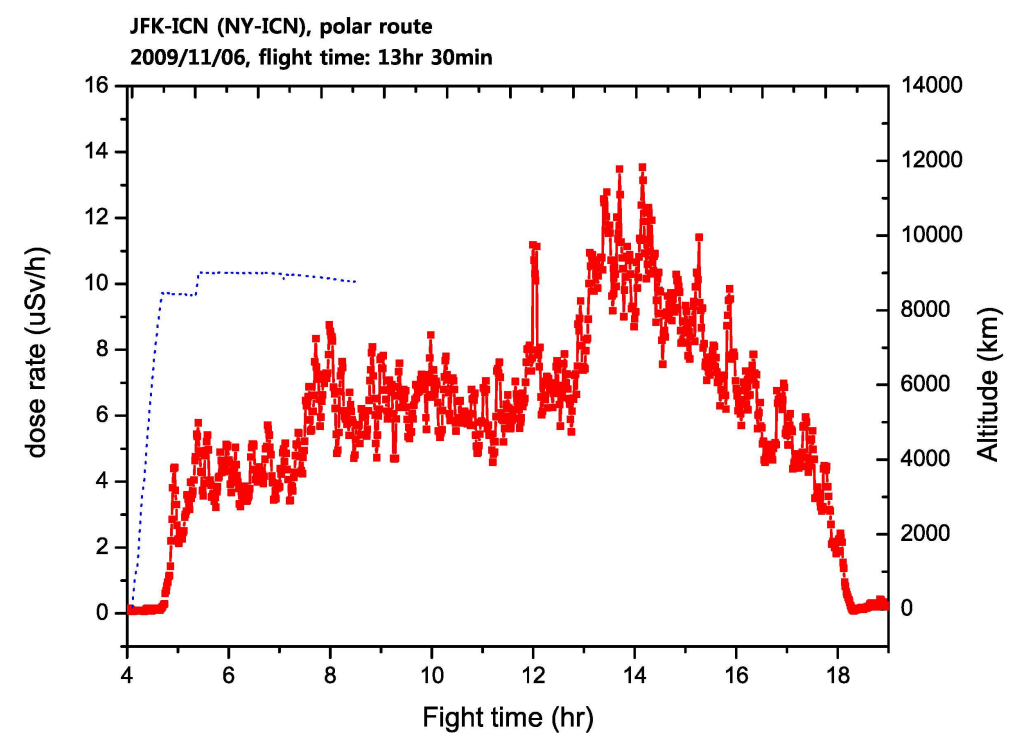

Figure 7. The third experiment the space radiation dose of the polar route (A airline).

Table 2. Results of the space radiation measurements and calculations for the polar route.

\begin{tabular}{ccccc}
\hline \hline Date & \multicolumn{2}{c}{ Total dose (uSv) } & $\begin{array}{c}\text { Average dose rate / STD } \\
(\mathrm{uSv} / \mathrm{hr})\end{array}$ & $\begin{array}{c}\text { Maximum dose rate } \\
\text { (uSv/h) }\end{array}$ \\
\cline { 2 - 3 } & Measure & CARI-6M & & $4.70 / 2.58$ \\
\hline $10 / 05$ & 76.07 & 63 & $4.75 / 3.76$ & 9.76 \\
$11 / 04$ & 91.84 & 75.3 & $6.36 / 10.81$ & 12.86 \\
$11 / 06$ & 86.48 & - & 5.27 & 13.54 \\
\hline Average & 84.79 & 69.15 & & 12.05 \\
\hline
\end{tabular}

due to declining altitude when arrival time to Incheon is only about 1 hour and 30 minutes remained.

Similarly, a result from the second polar route experiment is shown in Figure 6. At this time, measurement equipment was onboard a cargo aircraft (B747) of B airline in Korea, however, practical measurement on gamma-scout was unfeasible due to temporary equipment failure. Due to the nature of cargo aircrafts, which does not allow passengers aboard, so the aircraft can fly hogher altitude than other passenger jet. Actually the result shows that it flew higher than a regular aircraft up to around $12 \mathrm{~km}$, in comparison to regular aircraft maintained the neighborhood of $10 \mathrm{~km}$ at it's highest altitude (Figure 5). Despite shorten flight time of about 10 minutes, due to increased altitude, a total space radiation cumulative exposure in the second experiment was $91.84 \mathrm{uSv}$, displaying 15.77 uSv higher than that of the measured value obtained from the first polar route experiment. Average radiation exposure of total flight time was $4.75 \mathrm{uSv} / \mathrm{hr}$, and peak exposure at $12.86 \mathrm{uSv} / \mathrm{hr}$ - which is a very high result - as shown in 13 hours of flight time. The actual flight time when passing the polar circle is about 5 hours and 40 minutes, which is marked in thick gray solid lines, similar to Figure 5. The average radiation exposure during passing the polar route was $6.92 \mathrm{uSv} / \mathrm{hr}$, peak exposure at $12.86 \mathrm{uSv} / \mathrm{hr}$, and cumulative exposure dose at $40.57 \mathrm{uSv}$, account for $44 \%$ of the whole accumulation. These rates are similar to the rates shown in the first polar route experiment.

A result of the third experiment on the polar route is shown in Figure 7. At this time, altitude 


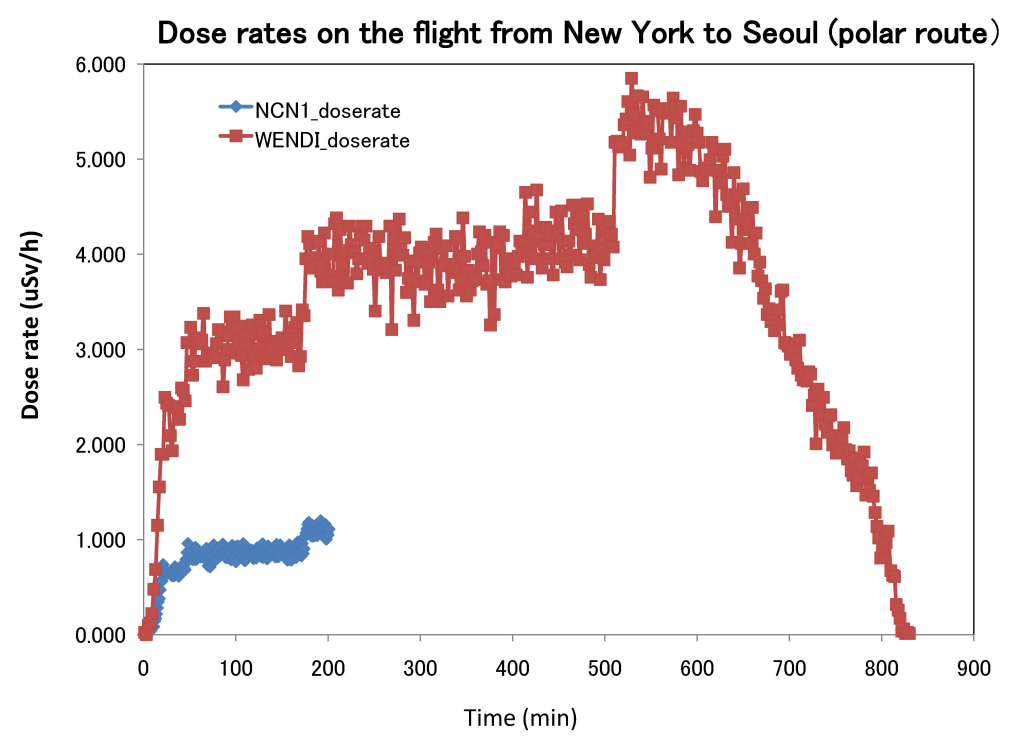

Figure 8. Results from NCN1 and WENDI of the third polar route experiment.

information showed in a discontinuity because location information was damaged due to GPS errors. Therefore, the calculation result of CARI-6M, which needs to input changed information of the precise location of latitude and altitude was unidentified. The total dose at this time was 86.48 uSv. Average exposure was $6.36 \mathrm{uSv} / \mathrm{hr}$, while peak exposure was $13.54 \mathrm{uSv} / \mathrm{hr}$, which is an extremely significant value. These can be presumed due to higher altitude however, the exact impact cannot be identified as the location information was lost. Dr. Hiroshi Yasuda of NIRS in Japan (National Institute of Radiological Sciences) has joined this third experiment, and this experiment was conducted concurrently with NCN1 and WENDI, space radiation measurement equipment for aircrafts procured by NIRS. Both NCN1 and WENDI are radiation measurement equipment reacting to neutrons. Since the response function of NCN1 reacts only to low neutron energy less than 10 $\mathrm{MeV}$, a measurement radiation value showed lower than that of WENDI, and the result is as Figure 8. The total dose of NCN1 was unverified as a measurement value was partly damaged due to mechanical errors of NCN1 during the experiment. Total exposure of WENDI showed $24.08 \mathrm{uSv}$. The reason for a difference in the total dose measured by Liulin-6K is that WENDI reacts to neutrons only, while Liulin-6K responses to proton, electron, and gamma rays, which eventually show such different results. Table 2 shows the result of the total three experiments on the polar route. As shown in Table 2, an average of total dose from the polar route is $84.79 \mathrm{uSv}$, and this is a higher value by $22 \%$ than $69.15 \mathrm{uSv}$, calculated by CARI-6M. One reason for such variation in calculated value and actual value could be attributed to inability to consider all elements of the space environment at that time.

Korean commercial flights are currently operating on the polar route using the north pacific route when depart, and selectively operate either on the polar route or the north pacific route at the time of returning only. That is, by considering space environment prediction at the time of departing, the polar route is not the best choice for flight when an extreme change is expected in a space environment, such as CME (Corona Mass Ejection) or Solar Flare, thus instead; a general route 


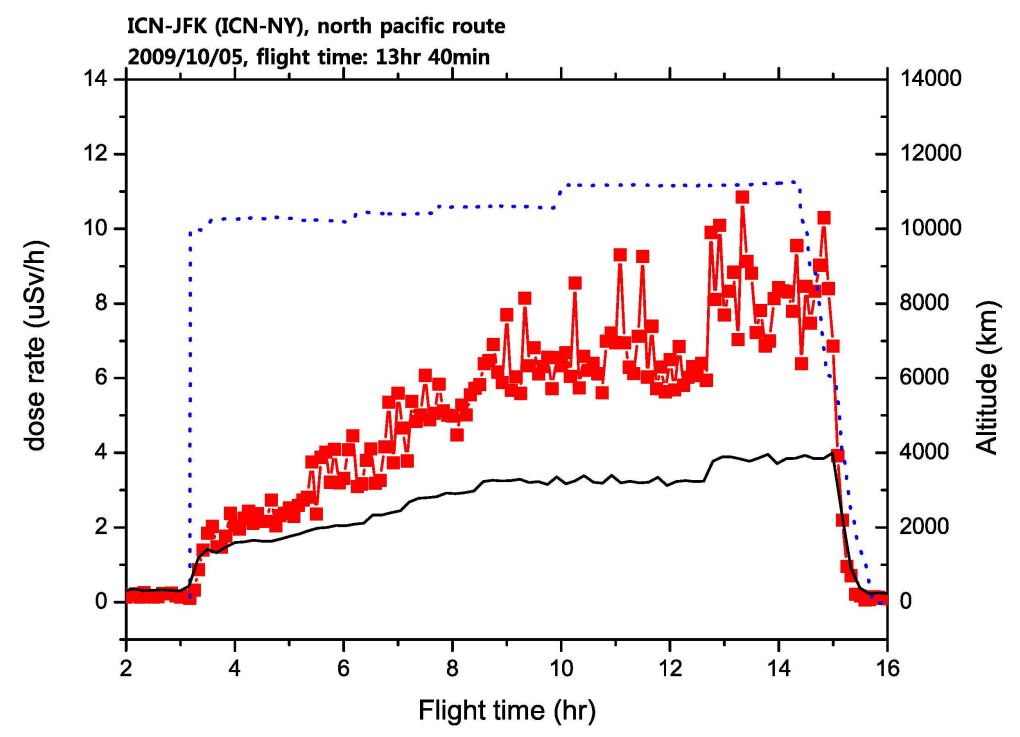

Figure 9. The first experiment measuring the space radiation dose of the north pacific route (A airline).

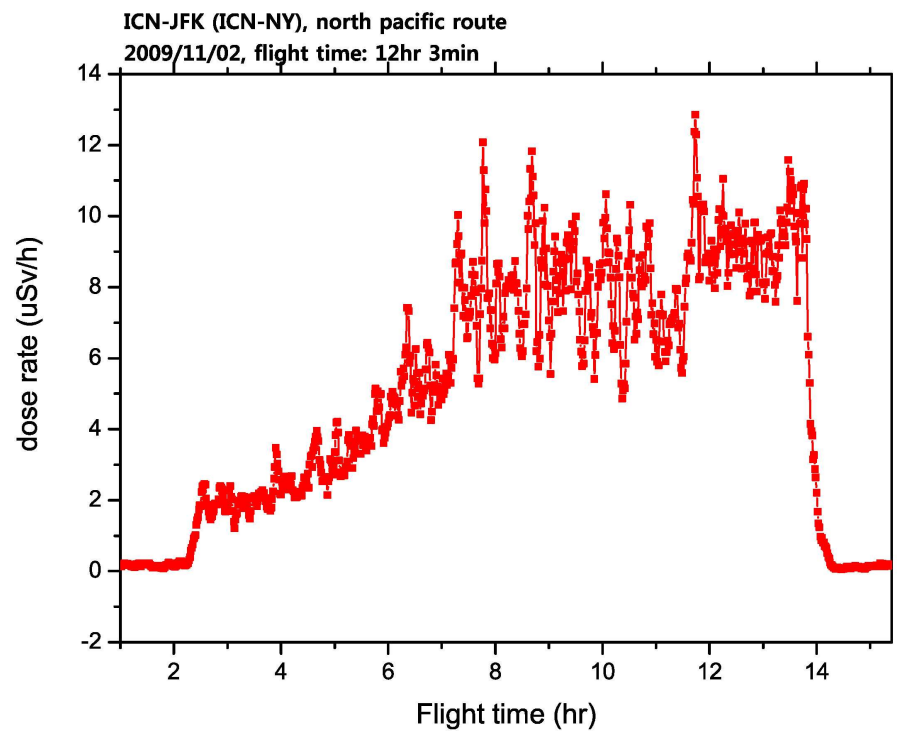

Figure 10. The second experiment measuring the space radiation dose of the north pacific route (B airline).

is used following FAAs regulations. In the experiments we conducted, the north pacific route was used for departing only, while the polar route was used for all returning flights. When considering a frequency of events, such as a solar burst, we assume that more returning flights operate on the polar route in general. 


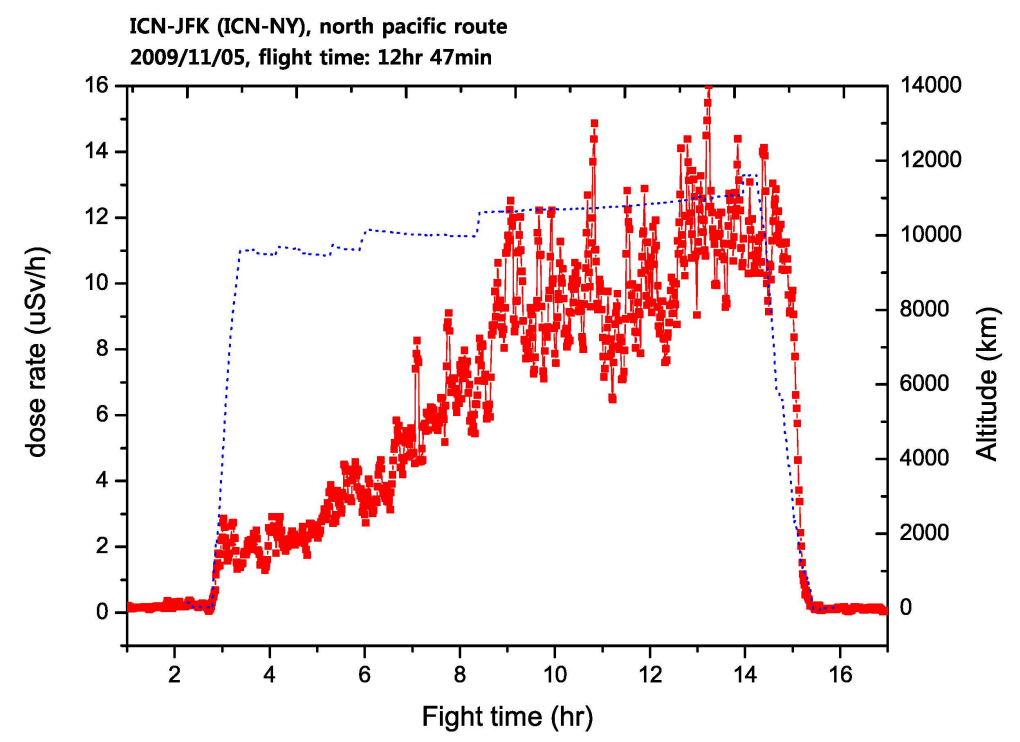

Figure 11. The third experiment measuring the space radiation dose of the north pacific route (A airline).

Table 3. Results of the space radiation measurements and calculations for the north pacific route.

\begin{tabular}{|c|c|c|c|c|}
\hline \multirow[t]{2}{*}{ Date } & \multicolumn{2}{|c|}{ Total dose (uSv) } & \multirow{2}{*}{$\begin{array}{c}\text { Average dose rate / STD } \\
(\mathrm{uSv} / \mathrm{hr})\end{array}$} & \multirow{2}{*}{$\begin{array}{c}\text { Maximum dose rate } \\
(\mathrm{uSv} / \mathrm{h})\end{array}$} \\
\hline & Measure & CARI-6M & & \\
\hline $10 / 05$ & 65.85 & 61.2 & $5.26 / 3.02$ & 10.85 \\
\hline $11 / 02$ & 65.83 & - & $4.14 / 4.79$ & 10.85 \\
\hline $11 / 05$ & 88.78 & - & $5.14 / 4.43$ & 16.14 \\
\hline Average & 73.48 & 61.2 & 4.84 & 12.61 \\
\hline
\end{tabular}

Table 4. Comparison of total dose between departure (non-polar route) and return flight (polar route).

\begin{tabular}{cccc}
\hline \hline Date & \multicolumn{2}{c}{ Total dose (uSv) } \\
\cline { 3 - 4 } & & Return (polar route) & Departure (north pacific route) \\
\hline $10 / 05$ & 76.07 & 65.85 \\
$11 / 02$ & 91.84 & 65.83 \\
$11 / 05$ & 86.48 & 88.78 \\
\hline Average & Measure & 84.7 & 73.48 \\
& CARI-6M & 84.9 cf: non-polar route 86.9 & 64.3 \\
\hline
\end{tabular}

Space radiation measurement similar to aforementioned has been conducted repeatedly for three times using the north pacific route at departure, and the result is shown in Figure 9, 10, and 11. Figure 9 shows results of space radiation from the north pacific route used at the time of departure on October 5 to conduct the first measurement experiment on the polar route, and the total dose was $65.85 \mathrm{uSv}$, average of $5.26 \mathrm{uSv} / \mathrm{hr}$, and peak of $10.85 \mathrm{uSv} / \mathrm{hr}$. As similar to Figure 5, the result measured via gamma-scout shows about $1 / 3$ less than the value measured by Liulin- $6 \mathrm{~K}$. The return flight's total dose was $76.07 \mathrm{uSv}$, a slightly higher compared to departing time using the north pacific route. Table 3 demonstrates results on departing flights using the north pacific route for three times. 


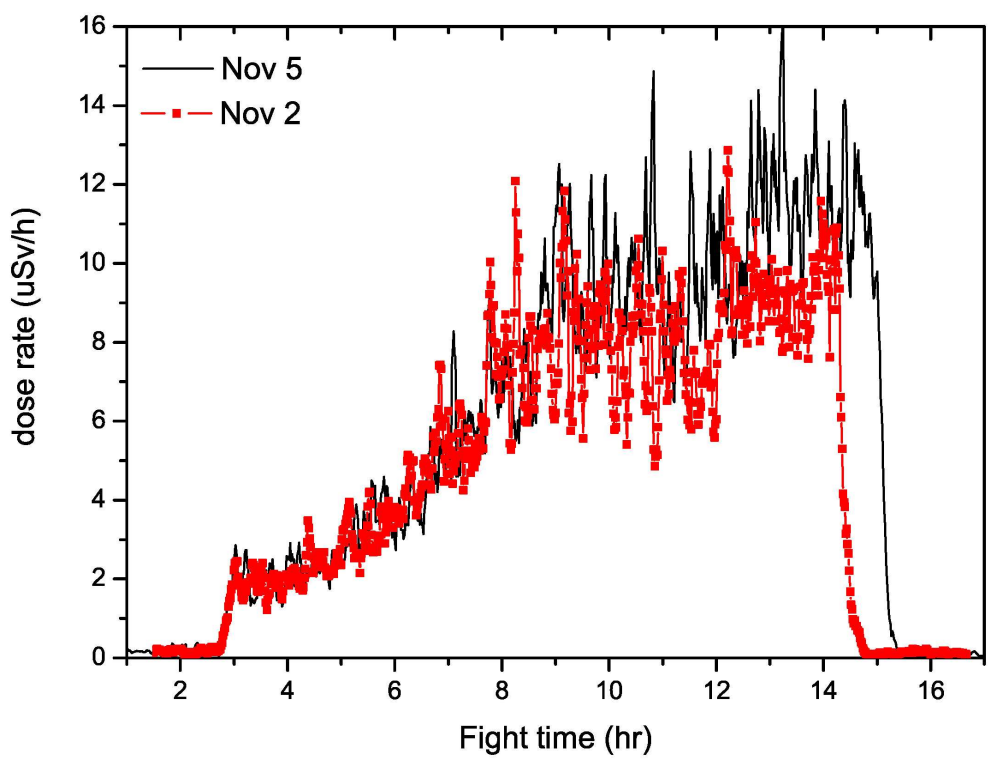

Figure 12. Comparison between the second and third experiments.

Table 4 illustrates a comparison of total dose on departure (the north pacific route) and returning (the polar route). As shown in Table 4, we verified that in general, the total radiation exposure at the time of returning using the polar route shows about $15 \%$ higher measurement compare to that of departure time. In the initial two experiments, total radiation exposure of the north pacific route at the departure time showed a value of around $65 \mathrm{uSv}$, which is similar to the value calculated using CARI-6M, however in the third experiment; the result was $88.78 \mathrm{uSv}$, which is $20 \mathrm{uSv}$ higher. For the cause analysis, Figure 12 is provided to show a comparison on exposure along with the actual altitude information. As shown in Figure 12, the third flight (November 5) showed about one hour longer than the second flight (November 2). These flight time differences seem to have eventually contributed in causing the difference of total cumulative radiation exposure. We presume that the third flight was higher in actual flight altitude as well, however, we regret not able to obtain an accurate comparison due to the loss of GPS data.

In Table 3, the measured value of total cumulative radiation exposure when returning was 84.7 uSv using the polar route, and the value measured by CARI-6M was $84.9 \mathrm{uSv}$, which is almost the same. Hence, we are confident to say that the calculation result of CARI-6M is reliable to some degree. In that case, it is necessary to compare the differences in radiation exposure in a case where the north pacific route, the non-polar route was used when returning, rather than the polar route, the route for returning flights. The total cumulative exposure calculated by CARI-6M using the north pacific route when returning is $86.9 \mathrm{uSv}$. That is, if an aircraft has returned using the north pacific route instead of the polar route, we can see that the effect of prolonged flight time is large enough to compensate the decreased space radiation due to lower latitude. Therefore, there is no need to be concerned about that a flight on the polar route on returning may be increasing exposure to space radiation, than using non-polar route. 


\section{Hwang et al.}

\section{Summary and Conclusion}

The study was performed as a policy assignment of the Office of Civil Aviation of the Ministry of Land, Transport and Maritime Affairs, which title is Developing safety standards and management policy of space radiation on the polar route". A process of space radiation measurement was conducted in the polar route and north pacific route by mounting space radiation measurement equipment, the first of its kind in Korea, in commercial flights. The measurement experiments on space radiation were conducted a total of six times using a B747 aircraft, which operates ICN-NY flights: Three experiments were conducted on the polar route and another three on north pacific route.

Average cumulative exposure on the polar route was $84.9 \mathrm{uSv}$, which is a value possible to fly about 70 times when annual dose limit is set to $6 \mathrm{mSv}$. Suppose the north pacific route is used when departing and the polar route when returning, it seems the conditions has to be considered when scheduling flight attendants and cabin attendants, as it can be reached in about 40 times of round trips. We found that there is no significant difference when flying to New York-Incheon in the same way using the polar route $(84.9 \mathrm{uSv})$ and the north pacific route $(86.9 \mathrm{uSv})$. This is determined that the space radiation exposure increased due to the flight on high latitudes using the polar routes is compensated by the one hour shorten flight time effect. Even if the same polar route is used, a typical average value should be determined by implementing a considerable number of practical measurement experiments, as we can see that there is some variation in the three measurement experiments conducted on the polar route (First: 76.07, Second: 91.84, and Third: $86.48 \mathrm{uSv}$ ). Therefore, space radiation measurement equipment should be onboard and implement long-term measurement experiments.

Acknowledgments: The study has been implemented with the support of "Development of Korean Space Weather Prediction Center" of Korea Astronomy and Space Science Institute. The research was implemented as a part of "Developing safety standards and management of space radiation on the polar route" effort of Office of Civil Aviation of the Ministry of Land, Transport and Maritime Affairs in 2009.

\section{References}

Cavallo, D., Marinaccio, A., Perniconi, B., Tomaoa, P., Pecoriello, V., Moccaldi, R., \& Iavicoli, S. 2002, Mutation Research, 513, 11

Green, A. R., Bennett, L. G. I., Lewis, B. J., Kitching, F., McCall, M. J., Desormeaux, M., \& Butler, A. 2005, Adv. Space. Res., 36, 1618

Lewis, B. J., Tume, P., Bennett, L. G. I., Pierre, M., Green, A. R., Cousins, T., Hoffarth, B. E., Jones, T. A., \& Brisson, J. R. 1999, Radiation Protection Dosimetry, 86, 7

Reynolds, P., Cone, J., Layefsky, M., Goldberg, D., \& Hurley, S. 2002, Cancer Causes and Control, 13,317

Spurny, F. \& Dachev, Ts. 2003, Adv. Space. Res., 32, 53

Spurny, F., Turek, K., VlcÏek, B., \& Dachev, Ts. 2004, Radiation Protection Dosimetry, 110, 1 